\title{
A new approach to Hardy-type inequalities
}

\author{
ADAM OSȨKOWSKI
}

\begin{abstract}
We introduce a new method which can be used to establish sharp Hardy-type inequalities on the positive halfline. As an illustration, we present a new proof of a classical result due to Bliss.
\end{abstract}

Mathematics Subject Classification. 58E35, 26A46.

Keywords. Hardy inequality, Best constants.

1. Introduction. A classical Hardy inequality states that for any nonnegative function $f$ on the positive halfline $(0, \infty)$, we have the sharp estimate

$$
\int_{0}^{\infty}\left(\frac{1}{t} \int_{0}^{t} f(s) \mathrm{d} s\right)^{k} \mathrm{~d} t \leq\left(\frac{k}{k-1}\right)^{k} \int_{0}^{\infty} f^{k}(t) \mathrm{d} t
$$

for any number $k>1$ (see e.g. Hardy, Littlewood, and Pólya [4]). Our motivation comes from the extension established by Hardy and Littlewood [3]:

$$
\int_{0}^{\infty} t^{\alpha}\left(\frac{1}{t} \int_{0}^{t} f(s) \mathrm{d} s\right)^{\ell} \mathrm{d} t \leq\left(\frac{k}{k-1}\right)^{k}\left(\int_{0}^{\infty} f^{k}(t) \mathrm{d} t\right)^{\ell / k},
$$

where $\ell \geq k>1$ and $\alpha=\ell / k-1$. Unfortunately, as Hardy and Littlewood observed, the constant $(k /(k-1))^{k}$ above is no longer optimal when $\ell>$ $k$. However, they guessed what the best value is, and their conjecture was confirmed a few years later by Bliss [1]. Here is the precise statement. 
Theorem 1.1. Suppose that $1<k<\ell$ are fixed, put $\alpha=\ell / k-1$, and let $f$ be a nonnegative function on $(0, \infty)$. Then we have the sharp bound

$$
\int_{0}^{\infty} t^{\alpha}\left(\frac{1}{t} \int_{0}^{t} f(s) d s\right)^{\ell} d t \leq C_{k, \ell, \alpha}\left(\int_{0}^{\infty} f^{k}(t) d t\right)^{\ell / k}
$$

where

$$
C_{k, \ell, \alpha}=\frac{1}{\ell-\alpha-1}\left[\frac{\alpha \Gamma(\ell / \alpha)}{\Gamma(1 / \alpha) \Gamma((\ell-1) / \alpha)}\right]^{\alpha} .
$$

Equipped with the above statement, one easily proves the following extension involving a power weight on the right hand side.

Theorem 1.2. Suppose that $1<k<\ell$ are fixed constants, and let $\alpha, \beta$ be positive numbers satisfying $\beta<k-1$ and $(1+\alpha)(1+\beta)^{-1}=\ell / k$. Then for any nonnegative function $f$ on $(0, \infty)$, we have

$$
\int_{0}^{\infty} t^{\alpha}\left(\frac{1}{t} \int_{0}^{t} f(s) d s\right)^{\ell} d t \leq C_{k, \ell, \alpha, \beta}\left(\int_{0}^{\infty} t^{\beta} f^{k}(t) d t\right)^{\ell / k},
$$

where

$$
C_{k, \ell, \alpha, \beta}=\frac{k(k-1)^{-\ell(k-1) / k}}{\ell(k-1-\beta)^{1-\ell(k-1) / k}}\left[\frac{\Gamma(\ell k /(\ell-k))}{\Gamma(\ell /(\ell-k)) \Gamma(k(\ell-1) /(\ell-k))}\right]^{\ell / k-1} .
$$

The inequality is sharp.

To see how this theorem follows from the previous one, simply apply (1.2) to the function $t \mapsto t^{\beta /(k-1-\beta)} f\left(t^{(k-1) /(k-1-\beta)}\right)$ and change the variables under the integrals.

There is a vast literature concerning various versions and applications of these results. It is impossible to review it here, and we refer the interested reader to the works [4-11] for an overview, history, and much more.

The main purpose of this paper is to introduce a novel method which can be used to prove general Hardy-type inequalities on the positive halfline. Roughly speaking, the method will allow to deduce a given estimate from the existence of a certain special function, possessing appropriate domination and monotonicity properties. In a sense, the technique is closely related to the so-called Bellman function method, a powerful tool used widely in harmonic analysis and probability theory: see e.g. [2,12-14] and the references therein. The method is described in the next section. In Section 3, we illustrate the technique by providing a novel proof of (1.2).

2. A method. Let $f$ be a nonnegative function on $(0,1]$, and let $k>1$ be fixed. Define

$$
X_{t}(f)=\frac{1}{t} \int_{0}^{t} f(x) \mathrm{d} x \quad \text { and } \quad Y_{t}(f)=\frac{1}{t} \int_{0}^{t} f^{k}(x) \mathrm{d} x
$$


for $t \in(0,1]$. A straightforward application of Hölder's inequality shows that the pair $\left(X_{t}, Y_{t}\right)$ takes values in the set

$$
\mathfrak{D}=\left\{(x, y) \in[0, \infty) \times[0, \infty): x^{k} \leq y\right\} .
$$

Next, let $V: \mathfrak{D} \times(0,1] \rightarrow[0, \infty), G: \mathfrak{D} \rightarrow[0, \infty)$ be two given Borel functions. Suppose that we are interested in showing the estimate

$$
\int_{0}^{1} V\left(X_{t}(f), Y_{t}(f), t\right) \mathrm{d} t \leq G\left(X_{1}(f), Y_{1}(f)\right)
$$

for all $f$. For instance, choose $V(x, y, t)=t^{\alpha} x^{\ell}$ and $G(x, y)=C y^{\ell / k}$ : then (2.2) becomes the "localized" Hardy inequality

$$
\int_{0}^{1} t^{\alpha-\ell}\left(\int_{0}^{t} f(s) \mathrm{d} s\right)^{\ell} \mathrm{d} t \leq C\left(\int_{0}^{1} f^{k}(s) \mathrm{d} s\right)^{\ell / k} .
$$

The key idea in the proof of $(2.2)$ is to consider a class $\mathcal{B}(V, G)$ which consists of all functions $B$ on $\mathfrak{D} \times(0,1]$ satisfying the following three properties.

i. (Nonnegativity) For any $(x, y, t) \in \mathfrak{D} \times(0,1]$ we have

$$
B(x, y, t) \geq 0 \text {. }
$$

ii. (Domination) For any $(x, y, t) \in \mathfrak{D} \times(0,1]$ we have

$$
B(x, y, 1) \leq G(x, y) \text {. }
$$

iii. (Monotonicity) For any nonnegative $f$ on $(0,1]$, the function

$$
\begin{aligned}
t \mapsto & B\left(X_{t}(f), Y_{t}(f), t\right)+\int_{t}^{1} V\left(X_{s}(f), Y_{s}(f), s\right) \mathrm{d} s \\
& \text { is nondecreasing on }(0,1] .
\end{aligned}
$$

Let us study the interplay between the class $\mathcal{B}(V, G)$ and the bound (2.2).

Lemma 2.1. If $\mathcal{B}(V, G)$ is nonempty, then the estimate (2.2) is valid.

Proof. For any nonnegative function $f$ on $(0,1]$, we have

$$
G\left(X_{1}(f), Y_{1}(f)\right) \geq B\left(X_{1}(f), Y_{1}(f), 1\right)
$$

by (2.4). Moreover, using the monotonicity property (iii), we may write

$$
\begin{aligned}
B\left(X_{1}(f), Y_{1}(f), 1\right) & =B\left(X_{1}(f), Y_{1}(f), 1\right)+\int_{1}^{1} V\left(X_{s}(f), Y_{s}(f), s\right) \mathrm{d} s \\
& \geq B\left(X_{t}(f), Y_{t}(f), t\right)+\int_{t}^{1} V\left(X_{s}(f), Y_{s}(f), s\right) \mathrm{d} s
\end{aligned}
$$

for any $t \in(0,1)$. Consequently, applying (2.3), we obtain 


$$
\int_{t}^{1} V\left(X_{s}(f), Y_{s}(f), s\right) \mathrm{d} s \leq G\left(X_{1}(f), Y_{1}(f)\right)
$$

for any $t$. Letting $t \rightarrow 0$ yields the claim, by virtue of Fatou's lemma.

A very interesting feature of the method is that the implication of the above lemma can be reversed. If the estimate (2.2) holds true, one can write an abstract (but non-explicit) formula for a special function $B$.

Lemma 2.2. If (2.2) is valid, then the class $\mathcal{B}(V, G)$ is nonempty.

Proof. For any $(x, y, t) \in \mathfrak{D} \times(0,1]$, introduce the class $\mathcal{M}(x, y, t)$ which consists of all nonnegative measurable functions $f$ on $(0, t]$ such that $\frac{1}{t} \int_{0}^{t} f(s) \mathrm{d} s=$ $x$ and $\frac{1}{t} \int_{0}^{t} f^{k}(s) \mathrm{d} s=y$. For each $(x, y, t)$, the class is nonempty: it contains, for instance, the function

$$
f(s)= \begin{cases}0 & \text { if } s \in(0, a), \\ t x /(t-a) & \text { if } s \in[a, t],\end{cases}
$$

where $a=\left(1-x^{k /(k-1)} / y^{1 /(k-1)}\right)$. Now, define a function $\mathbb{B}$ on $\mathfrak{D} \times(0,1]$ by

$$
\mathbb{B}(x, y, t)=\sup \left\{\int_{0}^{t} V\left(X_{s}(f), Y_{s}(f), s\right) \mathrm{d} s\right\},
$$

where the supremum is taken over all $f \in \mathcal{M}(x, y, t)$. Let us verify that $\mathbb{B}$ satisfies the required properties (2.3), (2.4), and (2.5). The first condition is evident since $V$ is nonnegative. The second property follows from (2.2): for any $f \in \mathcal{M}(x, y, 1)$ we have $\int_{0}^{1} V\left(X_{s}(f), Y_{s}(f), s\right) \mathrm{d} s \leq G(x, y)$. Thus, taking the supremum over all $f$ as above, we get (2.4). So, it remains to establish (2.5), for which the reasoning is slightly more complicated. Pick any function $f:(0,1] \rightarrow$ $[0, \infty)$ and fix $0 \leq t<u \leq 1$. Take an arbitrary $\tilde{f} \in \mathcal{M}\left(X_{t}(f), Y_{t}(f), t\right)$, and let us "splice" $f$ and $\tilde{f}$ into one function $\bar{f}=\tilde{f} \chi_{(0, t]}+f \chi_{(t, 1]}$. Observe that for any $r \in[t, 1]$,

$$
X_{r}(\bar{f})=\frac{1}{r} \int_{0}^{r} \bar{f} \mathrm{~d} s=\frac{1}{r}\left(\int_{0}^{t} \tilde{f} \mathrm{~d} s+\int_{t}^{r} f \mathrm{~d} s\right)=\frac{1}{r} \int_{0}^{r} f \mathrm{~d} s=X_{r}(f),
$$

where in the third equality we used the assumption $X_{t}(\tilde{f})=X_{t}(f)$. Similarly, one proves that $Y_{r}(\bar{f})=Y_{r}(f)$ for $r \in[t, 1]$. Denote $X_{t}=X_{t}(f), \bar{X}_{t}=X_{t}(\bar{f})$, $\tilde{X}_{t}=X_{t}(\tilde{f})$ and similarly for $Y_{t}, \bar{Y}_{t}$ and $\tilde{Y}_{t}$. So, by the definition of $\mathbb{B}$, we get

$$
\begin{aligned}
& \mathbb{B}\left(X_{u}, Y_{u}, u\right)+\int_{u}^{1} V\left(X_{s}, Y_{s}, s\right) \mathrm{d} s \\
& \geq \int_{0}^{u} V\left(\bar{X}_{s}, \bar{Y}_{s}, s\right) \mathrm{d} s+\int_{u}^{1} V\left(X_{s}, Y_{s}, s\right) \mathrm{d} s
\end{aligned}
$$




$$
\begin{aligned}
& =\int_{0}^{t} V\left(\bar{X}_{s}, \bar{Y}_{s}, s\right) \mathrm{d} s+\int_{t}^{u} V\left(\bar{X}_{s}, \bar{Y}_{s}, s\right) \mathrm{d} s+\int_{u}^{1} V\left(X_{s}, Y_{s}, s\right) \mathrm{d} s \\
& =\int_{0}^{t} V\left(\tilde{X}_{s}, \tilde{Y}_{s}, s\right) \mathrm{d} s+\int_{t}^{1} V\left(X_{s}, Y_{s}, s\right) \mathrm{d} s+\int_{u}^{1} V\left(X_{s}, Y_{s}, s\right) \mathrm{d} s \\
& =\int_{0}^{t} V\left(\tilde{X}_{s}, \tilde{Y}_{s}, s\right) \mathrm{d} s+\int_{t}^{1} V\left(X_{s}, Y_{s}, s\right) \mathrm{d} s .
\end{aligned}
$$

Taking the supremum over all $\tilde{f}$, we get the desired property (iii).

Suppose we have fixed $V, G$ and we want to prove (2.2) with the use of the above method. How to find an appropriate $B$ and how do we check that it has the desired properties? To address these issues, let us make a few comments on the properties $(2.3),(2.4)$, and (2.5). The first two of them are just appropriate dominations for the function $B$ : it can be neither too small nor too large. The most mysterious condition is the third one, and at the first glance it seems to be difficult to verify. Thus, it would be desirable to rephrase it in a more convenient form. We can offer a partial result in this direction. Namely, in many situations one can restrict oneself to showing (2.2) for continuous $f$ only; furthermore, the functions $V$ and $G$ are often smooth, and one can expect the desired $B$ to have this regularity as well. Note that

$$
\frac{\mathrm{d}}{\mathrm{d} t} X_{t}(f)=\frac{f(t)-X_{t}(f)}{t} \quad \text { and } \quad \frac{\mathrm{d}}{\mathrm{d} t} Y_{t}(f)=\frac{f^{k}(t)-Y_{t}(f)}{t}
$$

for any $t \in(0,1)$. So, a direct differentiation of the function in (2.5) leads to the following, slightly stronger form of (iii), which we state separately.

Lemma 2.3. Suppose that $B$ is continuous, of class $C^{1}$ in the interior of its domain, and satisfies

$$
B_{x}(x, y, t)\left(\frac{d}{t}-\frac{x}{t}\right)+B_{y}(x, y, t)\left(\frac{d^{k}}{t}-\frac{y}{t}\right)+B_{t}(x, y, t) \geq V(x, y, t)
$$

for any $(x, y, t) \in \mathfrak{D} \times(0,1)$ and $d \geq 0$. Then (iii) holds true.

This lemma also indicates how to search for $B$ : it is enough to construct a smooth function which satisfies (2.3), (2.4), and the above differential inequality (2.6). Though the latter still looks complicated, in many cases it can be handled due to some additional, "structural" properties of $B$ : see below.

Before we proceed, let us mention that the above method admits many extensions. We will give just one example, concerning bounds of the form (2.2) with other choices of the operators $X$. and $Y$; further modifications are easily found by the reader. Fix $\Phi:[0, \infty) \rightarrow[0, \infty), \beta \geq 0$ and let

$$
X_{t}(f)=\frac{1}{t} \int_{0}^{t} f(s) \mathrm{d} s \quad \text { and } \quad Y_{t}(f)=\frac{1}{t} \int_{0}^{t} s^{\beta} \Phi(f(s)) \mathrm{d} s .
$$


Let $\mathfrak{D}$ be the set in which $(X .(f), Y .(f))$ might take its values (it depends on $\Phi$ and $\beta)$, fix $V: \mathfrak{D} \times(0,1] \rightarrow[0, \infty), G: \mathfrak{D} \rightarrow[0, \infty)$ and suppose that we want to show $(2.2)$ for all nonnegative $f$ on $(0,1]$. Then, as one easily verifies, Lemmas 2.1 and 2.2 remain valid, but Lemma 2.3 needs to be slightly modified. With the above $X$. and $Y$, the stronger version of (iii) is

$$
B_{x}(x, y, t)\left(\frac{d}{t}-\frac{x}{t}\right)+B_{y}(x, y, t)\left(\frac{t^{\beta} \Phi(d)}{t}-\frac{y}{t}\right)+B_{t}(x, y, t) \geq V(x, y, t)
$$

for $(x, y, t) \in \mathfrak{D} \times(0,1]$. This is easily checked by the differentiation in $(2.5)$.

3. A new proof of Bliss' result. For the sake of clarity, we have decided to split this section into three parts.

3.1. Some special functions. We start with the following technical fact.

Lemma 3.1. For any $s \in(1, \infty)$ there is a unique $u=u(s) \in(0,1)$ which satisfies the identity

$$
\frac{(\alpha+1)(k-1)}{\alpha} \int_{u}^{1}(1-w)^{1 / \alpha} w^{(k-1) / \alpha+k-2} d w=\left(s-u^{k-1}\right)(1-u)^{1 / \alpha} u^{(k-1) / \alpha} .
$$

Furthermore, the function $u$ is of class $C^{1}$.

Proof. Let $s>1$ be fixed, and denote by $F(u)$ the difference of the left-hand side and the right-hand side of (3.1). Observe that $F^{\prime}(u)$ equals

$$
\begin{aligned}
- & \frac{(\alpha+1)(k-1)}{\alpha}(1-u)^{1 / \alpha} u^{(k-1) / \alpha+k-2}+(k-1) u^{k-2}(1-u)^{1 / \alpha} u^{(k-1) / \alpha} \\
& +\frac{s-u^{k-1}}{\alpha}(1-u)^{1 / \alpha-1} u^{(k-1) / \alpha}-\frac{k-1}{\alpha}\left(s-u^{k-1}\right)(1-u)^{1 / \alpha} u^{(k-1) / \alpha-1} .
\end{aligned}
$$

After some manipulations we check that $F^{\prime}(u)$ has the same sign as $G(u)=$ $\left(s-u^{k-1}\right) u-s(k-1)(1-u)$. Now, we see that $G(0)<0, G(u)$ is positive for $u$ sufficiently close to 1 , and $G^{\prime}(u)=k\left(s-u^{k-1}\right)>0$. So, there is $u_{0}$ such that $G$ is negative on $\left(0, u_{0}\right)$ and positive on $\left(u_{0}, 1\right)$, and hence, $F$ is decreasing on $\left(0, u_{0}\right)$ and increasing on $\left(u_{0}, 1\right)$. But we easily check that $F(0)>0$ and $F(1)=0$; thus $F$ has a unique zero inside $(0,1)$. The claimed regularity of the function $u$ follows at once from the well-known facts on implicit functions.

Lemma 3.2. There is an increasing function $\varphi:[1, \infty) \rightarrow \mathbb{R}$ satisfying

$$
(k-1)\left[1-\left(\frac{s \varphi^{\prime}(s)-\frac{\ell}{k} \varphi(s)}{\varphi^{\prime}(s)}\right)^{1 /(k-1)}\right]\left(s \varphi^{\prime}(s)-\frac{\ell}{k} \varphi(s)\right)=1
$$

for $s \in(1, \infty)$ and the initial condition $\varphi(1)=k / \ell$.

Proof. Let $u$ be the function introduced in the previous lemma, and put

$$
\varphi(s)=\frac{k}{\ell(k-1)} \frac{s-u^{k-1}(s)}{(1-u(s)) u^{k-1}(s)} .
$$


We compute that (for brevity, we have decided to omit the argument $s$ and write $u$ instead of $u(s), \varphi$ instead of $\varphi(s)$, etc.)

$$
\varphi^{\prime}=\frac{k}{\ell(k-1)}\left[\frac{1-(k-1) u^{k-2} u^{\prime}}{(1-u) u^{k-1}}+\frac{s-u^{k-1}}{(1-u)^{2} u^{k-1}} u^{\prime}-\frac{(k-1)\left(s-u^{k-1}\right) u^{\prime}}{(1-u) u^{k}}\right] \text {, }
$$

which implies

$$
\begin{aligned}
& (k-1)(1-u) u^{k-1} \varphi^{\prime} \\
& \quad=\frac{k}{\ell}\left[1-(k-1) u^{k-2} u^{\prime}+\frac{s-u^{k-1}}{1-u} u^{\prime}-\frac{(k-1)\left(s-u^{k-1}\right) u^{\prime}}{u}\right] .
\end{aligned}
$$

Now differentiate (3.1) with respect to $s$, the argument of $u$, to get

$$
\begin{aligned}
& -\frac{(\alpha+1)(k-1)}{\alpha}(1-u)^{1 / \alpha} u^{(k-1) / \alpha+k-2} u^{\prime} \\
& =\left(1-(k-1) u^{k-2} u^{\prime}\right)(1-u)^{1 / \alpha} u^{(k-1) / \alpha}-\frac{s-u^{k-1}}{\alpha}(1-u)^{1 / \alpha-1} u^{(k-1) / \alpha} u^{\prime} \\
& \quad+\frac{k-1}{\alpha}\left(s-u^{k-1}\right)(1-u)^{1 / \alpha} u^{(k-1) / \alpha-1} u^{\prime},
\end{aligned}
$$

or equivalently,

$$
-\frac{\ell(k-1)}{k} u^{k-2} u^{\prime}=\alpha\left[1-(k-1) u^{k-2} u^{\prime}\right]-\frac{s-u^{k-1}}{1-u} u^{\prime}+(k-1) \frac{\left(s-u^{k-1}\right) u^{\prime}}{u} .
$$

Plug this into (3.4): then the right-hand side becomes 1 , so we get

$$
\varphi^{\prime}=\frac{1}{(k-1)(1-u) u^{k-1}}=\frac{\ell}{k} \frac{\varphi}{s-u^{k-1}},
$$

which, in turn, implies

$$
u=\left(\frac{s \varphi^{\prime}(s)-\frac{\ell}{k} \varphi(s)}{\varphi^{\prime}(s)}\right)^{1 /(k-1)} .
$$

Inserting this identity into (3.2) transforms it into (3.5); this shows that $\varphi$ [given by (3.3)] enjoys the differential equation (3.2), as desired. The monotonicity of $\varphi$ follows immediately from (3.5). It remains to show that this $\varphi$ satisfies $\lim _{s \downarrow 1} \varphi(s)=k / \ell$. To do this, observe that $\lim _{s \rightarrow 1} u(s)=1$, by the very definition of $u$, and consequently, using (3.1),

$$
\begin{aligned}
\varphi & =\frac{k}{\ell} \frac{s-u^{k}}{(k-1)(1-u) u^{k-1}} \\
& =\frac{k}{\ell} \frac{\alpha+1}{\alpha} \frac{u^{-k / \alpha-k}}{(1-u)^{1 / \alpha+1}} \int_{u}^{1}(1-w)^{1 / \alpha} w^{(k-1) / \alpha+k-2} \mathrm{~d} w \stackrel{s \downarrow 1}{\longrightarrow} \frac{k}{\ell},
\end{aligned}
$$

by de l'Hospital rule. This completes the proof.

The final property of $\varphi$ is the following boundedness condition.

Lemma 3.3. Let $C_{k, \ell, \alpha}$ denote the constant (1.3). For any $s \geq 1$,

$$
\varphi(s) \leq C_{k, \ell, \alpha} s^{\ell / k} \text {. }
$$


Proof. By (3.6), for any $s>1$ we have

$$
\left[\frac{\varphi(s)}{s^{\ell / k}}\right]^{\prime}=s^{-1-\ell / k}\left(s \varphi^{\prime}(s)-\frac{\ell}{k} \varphi(s)\right)=s^{-1-\ell / k} \varphi^{\prime}(s) u(s)^{k-1}>0,
$$

so we will be done if we manage to establish the identity $\lim _{s \rightarrow \infty} \varphi(s) s^{-\ell / k}=$ $C_{k, \ell, \alpha}$. To do this, note first that by (3.1), (3.3), and (3.5),

$$
\begin{aligned}
& \frac{(\alpha+1)(k-1)}{\alpha} \int_{u}^{1}(1-w)^{1 / \alpha} w^{(k-1) / \alpha+k-2} \mathrm{~d} w \\
& =\frac{s-u^{k-1}}{(1-u) u^{k-1}} \cdot(1-u)^{1 / \alpha+1} u^{(k-1) / \alpha+k-1} \\
& =\frac{\ell(k-1)}{k} \varphi(s) \cdot \frac{1}{(k-1)^{1 / \alpha+1}\left(\varphi^{\prime}(s)\right)^{1 / \alpha+1}} .
\end{aligned}
$$

This implies

$$
\lim _{s \rightarrow \infty} \frac{\varphi(s)}{\left(\varphi^{\prime}(s)\right)^{1 / \alpha+1}}=\frac{k(k-1)^{1 / \alpha+1}}{\ell-k} \int_{0}^{1}(1-w)^{1 / \alpha} w^{(k-1) / \alpha+k-2} \mathrm{~d} w,
$$

or, which is the same,

$\lim _{s \rightarrow \infty} \frac{\varphi^{\prime}(s)}{\varphi(s)^{\alpha /(\alpha+1)}}=\left[\frac{k(k-1)^{1 / \alpha+1}}{\ell-k} \int_{0}^{1}(1-w)^{1 / \alpha} w^{(k-1) / \alpha+k-2} \mathrm{~d} w\right]^{-\alpha /(\alpha+1)}$.

A little calculation shows that the right-hand side is equal to $C_{k, \ell, \alpha}^{1 /(\alpha+1)}$. Therefore, if $s_{0}>1$ is a fixed number and $s>s_{0}$ is arbitrary, we may write

$$
\begin{aligned}
\frac{\varphi(s)}{s^{\ell / k}}=\left[\frac{\varphi^{1 /(\alpha+1)}(s)}{s}\right]^{\alpha+1} & =\left[\frac{\varphi^{1 /(\alpha+1)}\left(s_{0}\right)+\int_{s_{0}}^{s} \frac{(\alpha+1)^{-1} \varphi^{\prime}(r)}{\varphi(r)^{\alpha /(\alpha+1)}} \mathrm{d} r}{s}\right]^{\alpha+1} \\
& \stackrel{s \rightarrow \infty}{\longrightarrow}\left(\lim _{s \rightarrow \infty} \frac{(\alpha+1) \varphi^{\prime}(s)}{\varphi(s)^{\alpha /(\alpha+1)}}\right)^{\alpha+1}=C_{k, \ell, \alpha} .
\end{aligned}
$$

3.2. Proof of (1.2). Fix $\ell, k$ and let $\alpha=\ell / k-1$. By an easy dilation, it is enough to prove that

$$
\int_{0}^{1} t^{\alpha-\ell}\left(\int_{0}^{t} f(s) \mathrm{d} s\right)^{\ell} \mathrm{d} t \leq C_{k, \ell, \alpha}\left(\int_{0}^{1} f^{k}(t) \mathrm{d} t\right)^{\ell / k}
$$

for any continuous nonnegative function $f$ on $[0,1]$. This bound is of the form (2.2), with $V(x, y, t)=t^{\alpha} x^{\ell}$ and $G(x, y)=C_{k, \ell, \alpha} y^{\ell / k}$. Thus we can apply the method of Section 2. As we will see, the right choice for $B$ is given by

$$
B(x, y, t)=t^{1+\alpha} x^{\ell} \varphi\left(x^{-k} y\right),
$$

where $\varphi$ is the function of Lemma 3.2. Clearly, this function is nonnegative and, by $(3.7)$, we have $B(x, y, 1)=x^{\ell} \varphi\left(x^{-k} y\right) \leq C_{k, \ell, \alpha} y^{\ell / k}=G(x, y)$. So, the 
conditions (2.3) and (2.4) are satisfied, and we need to verify (2.5). By Lemma 2.3 and the reasoning preceding it, we will be done if we check that $B$ satisfies (2.6). By the direct differentiation of $B$, this is equivalent to

$$
\begin{aligned}
& k t^{1+\alpha} x^{\ell-1}\left(\frac{\ell}{k} \varphi(s)-s \varphi^{\prime}(s)\right)\left(\frac{d}{t}-\frac{x}{t}\right)+t^{1+\alpha} x^{\ell-k} \varphi^{\prime}(s)\left(\frac{d^{k}}{t}-\frac{y}{t}\right) \\
& \quad+(1+\alpha) t^{\alpha} x^{\ell} \varphi(s) \geq t^{\alpha} x^{\ell},
\end{aligned}
$$

where $s=y / x^{k}$. If we divide both sides by $x^{\ell} t^{\alpha}$, the bound becomes

$$
k\left(\frac{\ell}{k} \varphi(s)-s \varphi^{\prime}(s)\right)(\xi-1)+\varphi^{\prime}(s)\left(\xi^{k}-s\right)+(1+\alpha) \varphi(s) \geq 1,
$$

where $\xi=d / x \geq 0$. By the previous subsection, we know that $\varphi^{\prime}(s)>0$ for all $s$. So, the left-hand side, considered as a function of $\xi$, attains its minimum for $\xi$ satisfying $k\left(\frac{\ell}{k} \varphi(s)-s \varphi^{\prime}(s)\right)+k \varphi^{\prime}(s) \xi^{k-1}=0$, or $\xi=\left(s-\frac{\ell}{k} \varphi(s) / \varphi^{\prime}(s)\right)^{1 /(k-1)}$. But for this $\xi$ both sides of (3.9) are equal: indeed,

$$
\begin{aligned}
& k\left(\frac{\ell}{k} \varphi(s)-s \varphi^{\prime}(s)\right)(\xi-1)+\varphi^{\prime}(s)\left(\xi^{k}-s\right)+(1+\alpha) \varphi(s)-1 \\
& \quad=(k-1)\left[1-\left(\frac{s \varphi^{\prime}(s)-\frac{\ell}{k} \varphi(s)}{\varphi^{\prime}(s)}\right)^{1 /(k-1)}\right]\left(s \varphi^{\prime}(s)-\frac{\ell}{k} \varphi(s)\right)-1,
\end{aligned}
$$

which is zero, due to (3.2). This proves that $B$ belongs to the class $\mathcal{B}(V, G)$.

3.3. On the search for $B$ and the optimality of $C_{k, \ell, \alpha}$. Let us sketch steps which led us to the discovery of the function $B$. As we have already observed above, the inequality (1.2) follows once we have established the localized bound (3.8), which is of the form (2.2), with $V(x, y, t)=t^{\alpha} x^{\ell}$ and $G(x, y)=C_{k, \ell, \alpha} y^{\ell / k}$. Write down the corresponding special function $\mathbb{B}$ :

$$
\mathbb{B}(x, y, t)=\sup \left\{\int_{0}^{t} s^{\alpha}\left(\frac{1}{s} \int_{0}^{s} f(r) \mathrm{d} r\right)^{\ell} \mathrm{d} s\right\},
$$

where the supremum is taken over all $f \in \mathcal{M}(x, y, t)$. Let us infer some "structural" properties of $\mathbb{B}$, directly from this definition. Observe that $f \in \mathcal{M}(x, y, t)$ if and only if the dilated function $\delta_{t} f: s \mapsto f(t s)$ belongs to $\mathcal{M}(x, y, 1)$. By direct substitutions in the integrals defining $\mathbb{B}(x, y, t)$, we get

$$
\mathbb{B}(x, y, t)=t^{1+\alpha} \sup \left\{\int_{0}^{1}\left(\frac{1}{s} \int_{0}^{s} \delta_{t} f(r) \mathrm{d} r\right)^{k} \mathrm{~d} s\right\}=t^{1+\alpha} \mathbb{B}(x, y, 1) .
$$

The next property comes from the observation that for any $\lambda>0$, we have $f \in \mathcal{M}(x, y, 1)$ if and only if $\lambda f \in \mathcal{M}\left(\lambda x, \lambda^{k} y, 1\right)$. So, by the definition of $\mathbb{B}$, we get $\mathbb{B}\left(\lambda x, \lambda^{k} y, 1\right)=\lambda^{\ell} \mathbb{B}(x, y, 1)$. Hence, taking $\lambda=1 / x$, we get $\mathbb{B}(x, y, t)=$ 
$t^{1+\alpha} \mathbb{B}(x, y, 1)=t^{1+\alpha} x^{\ell} \mathbb{B}\left(1, \frac{y}{x^{k}}, 1\right)$ for all $(x, y, t)$. Putting $\varphi(s)=\mathbb{B}(1, s, 1)$, we get the explanation for the special form of the function $B$ used above. How can we find $\varphi$ ? The indication is contained in the structure of the above proof. Namely, the work with the condition of Lemma 2.3 leads us to (3.9), and the further optimization over $\xi$ shows that the minimum, given by (3.10), should be nonnegative. Assuming that this minimum is zero for all $s$, we obtain the differential equation (3.2). To get the initial value $\varphi(1)=\mathbb{B}(1,1,1)$, note that the class $\mathcal{M}(1,1,1)$ contains only one element (up to a set of Lebesgue measure 0 ): the constant function $f \equiv 1$. Plugging this function into the definition of $\mathbb{B}(1,1,1)$, we get that this value must be $\ell / k$, and hence $\varphi(1)=\ell / k$. This gives us the above special function $\varphi$. Finally, the constant $C_{k, \ell, \alpha}$ is just the smallest number such that the inequality (3.7) holds true.

The above approach also gives an indication about the extremal functions in (3.8) (i.e., about the functions for which the equality, or almost equality, is attained). We would like to stress here that the arguments presented below will not be strict, as their goal is to lead us to the right guess for the extremal functions. The idea is very simple: we want to find an $f$ such that all the intermediate inequalities appearing during the application of the method become equalities. Let us be more specific. As we already know, for a given smooth $f$ : $(0,1] \rightarrow[0, \infty)$, the function $t \mapsto B\left(X_{t}(f), Y_{t}(f), t\right)+\int_{t}^{1} V\left(X_{s}(f), Y_{s}(f), s\right) \mathrm{d} s$ is nondecreasing. This, by the direct differentiation, gives (3.9) with the parameters $s=Y_{t}(f) / X_{t}^{k}(f)$ and $\xi=f(t) / X_{t}(f)$. Then the optimization over $\xi$ leads to (3.2): the extremal choice for $\xi$ is $\left(s-\frac{\ell}{k} \varphi(s) / \varphi^{\prime}(s)\right)^{1 /(k-1)}$, as we already computed above. Thus, it is natural to suspect that the extremal function $f$ satisfies the equality

$$
\xi=\xi(t)=\frac{f(t)}{X_{t}(f)}=\left(s-\frac{\ell}{k} \frac{\varphi(s)}{\varphi^{\prime}(s)}\right)^{1 /(k-1)}=u(s)
$$

for all $t \in(0,1]$, where $s=Y_{t}(f) / X_{t}^{k}(f)$. Plugging this into (3.1), we obtain

$$
\begin{aligned}
& \frac{(\alpha+1)(k-1)}{\alpha} \int_{f(t) / X_{t}(f)}^{1}(1-w)^{1 / \alpha} w^{(k-1) / \alpha+k-2} \mathrm{~d} w \\
& =\left(\frac{Y_{t}(f)}{X_{t}^{k}(f)}-\frac{f^{k-1}(t)}{X_{t}^{k-1}(f)}\right)\left(1-\frac{f(t)}{X_{t}(f)}\right)^{1 / \alpha}\left(\frac{f(t)}{X_{t}(f)}\right)^{(k-1) / \alpha} .
\end{aligned}
$$

Assuming that $f$ is smooth, let us differentiate both sides with respect to $t$. We obtain an equality equivalent to $I+I I=0$, where

$$
\begin{aligned}
& I=\left(\frac{Y_{t}(f)}{X_{t}^{k}(f)}-\frac{f^{k-1}(t)}{X_{t}^{k-1}(f)}\right)\left(\xi^{\prime}(t)+\frac{\alpha \xi(t)(1-\xi(t))}{t}\right) \frac{k-1-k \xi(t)}{\alpha}, \\
& I I=\alpha^{-1}(k-1) \xi^{k-1}(t)(1-\xi(t))\left(\xi^{\prime}(t)+\frac{\alpha \xi(t)(1-\xi(t))}{t}\right) .
\end{aligned}
$$

We see that the factor $\xi^{\prime}(t)+\alpha \xi(t)(1-\xi(t)) / t$ is present in both terms: thus, let us find $f$ for which it vanishes. The general solution to the differential equation $\xi^{\prime}(t)=-\alpha \xi(t)(1-\xi(t)) / t, t \in(0,1)$, is given by $\xi(t)=\left(1+d t^{\alpha}\right)^{-1}$, as one 
easily proves by the separation of variables. Here $d$ is an arbitrary constant. Hence, from the definition of $\xi$, we get $f(t)=\left(1+d t^{\alpha}\right)^{-1} X_{t}(f)$, or, using the identity $f(t)=t \frac{d}{d t} X_{t}(f)+X_{t}(f)$,

$$
\frac{\mathrm{d}}{\mathrm{d} t} X_{t}(f)=\frac{X_{t}(f)}{t\left(1+d t^{\alpha}\right)}-\frac{X_{t}(f)}{t} .
$$

This equation is also easy to solve: the general solution is $X_{t}(f)=c(1+$ $\left.d t^{\alpha}\right)^{-1 / \alpha}$, where $c$ is an arbitrary constant. This yields the following candidate for the extremizer: $f(t)=c\left(1+d t^{\alpha}\right)^{-(1+\alpha) / \alpha}$. This is indeed the right choice: letting $d \rightarrow \infty$ and carrying out some easy manipulations, we see that

$$
\frac{\int_{0}^{1} t^{\alpha}\left(\frac{1}{t} \int_{0}^{t} f(s) \mathrm{d} s\right)^{\ell} \mathrm{d} t}{\left(\int_{0}^{1} f^{k}(t) \mathrm{d} t\right)^{\ell / k}} \rightarrow \frac{\int_{0}^{\infty} t^{\alpha}\left(1+t^{\alpha}\right)^{-\ell / \alpha} \mathrm{d} t}{\int_{0}^{\infty}\left(1+t^{\alpha}\right)^{-k(1+\alpha) / \alpha} \mathrm{d} t}=C_{k, \ell, \alpha} .
$$

We conclude the paper with the following comment.

Remark 3.4. There is a natural question about other Hardy-type inequalities which can be successfully treated with the above method. As an example (a partial answer to this question), one can show that the estimate of Theorem 1.2 can be proved with the use of a certain special function $B$. However, we have decided not to include any details here, since, as we have noted in the introductory section, the result follows at once from Theorem 1.1.

Acknowledgement. The author would like to thank an anonymous referee for the careful reading of the first version of the manuscript and several helpful comments.

Open Access. This article is distributed under the terms of the Creative Commons Attribution License which permits any use, distribution, and reproduction in any medium, provided the original author(s) and the source are credited.

\section{References}

[1] G. Bliss, An integral inequality, J. London. Math. Soc. 5 (1930), 40-46.

[2] D. L. Burkholder, Explorations in martingale theory and its applications, École d'Eté de Probabilités de Saint-Flour XIX - 1989, pp. 1-66, Lecture Notes in Math., 1464, Springer, Berlin, 1991.

[3] G. H. Hardy and J. E. Littlewood, Notes on the theory of series (XII): On certain inequalities connected with calculus of variations, J. London Math. Soc. 5 (1930), 34-39.

[4] G. H. Hardy, J. E. Littlewood, and G. Pólya, Inequalities, 2nd edn. (Cambridge University Press, 1952).

[5] A. Kufner, L. Maligranda, And L. E. Persson, The Prehistory of the Hardy Inequality, Amer. Math. Monthly, 113 (2006), 715-732.

[6] A. Kufner, L. Maligranda, And L. E. Persson, The Hardy inequality. About its history and some related results, Vydavatelský Servis, Plzeň, 2007. 162 pp. 
[7] A. Kufner, And B. OpIC, Hardy-type inequalities, Longman Scientific and Technical, Harlow, 1990.

[8] A. Kufner, And L. E. Persson, Weighted Inequalities of Hardy Type, World Sci., River Edge, NJ, 2003.

[9] N. Masmoudi, About the Hardy inequality, An invitation to Mathematics, pp. 165-180 (2011), Springer Berlin Heidelberg.

[10] V. G. MazÝA, Sobolev spaces, Springer-Verlag, Berlin, 1985.

[11] D. S. Mitrinovic, J. E. Pecaric, And A. M. Fink, Inequalities Involving Functions and Their Derivatives, Kluwer Acad. Publishers, Dordrecht-BostonLondon, 1991.

[12] F. L. Nazarov and S. R. Treil, The hunt for a Bellman function: applications to estimates for singular integral operators and to other classical problems of harmonic analysis, St. Petersburg Math. J. 8 (1997), 721-824.

[13] A. Oş̧Kowski, Sharp martingale and semimartingale inequalities, Monografie Matematyczne 72 (2012), Birkhäuser Basel.

[14] V. Vasyunin, A. Volberg, Bellman functions technique in Harmonic Analysis, http://sashavolberg.wordpress.com

Adam OsȨKOWsKI

Faculty of Mathematics,

Informatics and Mechanics,

University of Warsaw,

Banacha 2, 02-097 Warsaw,

Poland

e-mail: ados@mimuw.edu.pl

Received: 26 June 2014 